com registo de avanço mandibular de 75,8 \pm 7,9\% de protrusão máxima. Os pacientes foram classificados como 'mau candidato", "candidato razoável" e "bom candidato" consoante a resolução do/os colapso/os da via aérea superior com a utilização do registo de avanço mandibular. De seguida, esses pacientes iniciaram o tratamento com DAM. Foram avaliados os colapsos da via aérea superior durante a DISE com registo de avanço mandibular e o resultado do tratamento com DAM. A resposta ao tratamento foi definida como uma redução no IAH, avaliada pelo traçado poligráfico do sono nível II em ambulatório, após 1 mês de tratamento com DAM para avaliar uma redução de pelo menos 50\% do IAH inicial. Resultados: Nos casos analisados 2 pacientes foram classificados como 'bom candidato' e os outros 2 como "candidato razoável". Relativamente à terapêutica instituída todos os candidatos apresentaram sucesso terapêutico com o DAM, ou seja, todos apresentaram uma diminuição do IAH > 50\% (IAH inicial $=20,2 \pm$ $6,8 / \mathrm{h}$ vs IAH após 1 mês de utilização de DAM = 5,2 $\pm 4 / \mathrm{h}$ ). Conclusões: Os resultados deste estudo sugerem que o uso de um registo de avanço mandibular em 75,8 \pm 7,9\% de protrusão máxima da mandíbula, nos indivíduos do estudo, durante uma DISE é mais um fator importante na previsão da resposta à terapêutica com DAM na SAOS.

http://doi.org/10.24873/j.rpemd.2022.01.908

\#052 Influência do matiz na eficácia do branqueamento dentário - Estudo clínico aleatorizado

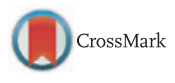

Ruben Pereira*, João Silveira, Susana Dias, Ana Cardoso, António Mata, Duarte Marques

Grupo de Investigação em Biologia e Bioquímica Oral (GIBBO) - LIBPhys FCT UID/FIS/04559/2013 da Faculdade de Medicina Dentária da Universidade de Lisboa

Objetivos: Avaliar o efeito do matiz na eficácia do branqueamento dentário por três técnicas diferentes. Materiais e métodos: Quarenta e cinco pacientes foram selecionados, por critérios de inclusão/exclusão pré-estabelecidos, para um ensaio clínico aleatorizado com três grupos: Grupo 1 - 6\% peróxido de hidrogénio em consultório (VivaStyle ${ }^{\circledR}$ Paint On Plus, IvoclarVivadent, Liechtenstein); Grupo 2- 6\% peróxido de hidrogénio em ambulatório com moldeira adaptável (Opalescence ${ }^{\circledR}$ GO, Ultradent, EUA); Grupo 3 - 16\% peróxido de carbamida em ambulatório com moldeira individual (Opalescence ${ }^{\circledR}$ PF, Ultradent, EUA). Foram realizados os protocolos de branqueamento de acordo com indicações do fabricante e registada a cor dentária, em valores CIE L*a*b* e na escala VITA Classical (VITA Zahnfabrick, Germany), dos 12 dentes anteriores, por um espectrofotómetro (SpectroShade, MHT Optic Research AG, Suiça), no início e final do tratamento. A diferença de cor $(\triangle \mathrm{E} 00)$ por sub-grupo de matiz (A, B, C e D) foi calculada no final do tratamento e apresentada como média e 95\% intervalo de confiança. Os testes one-way e two-way ANOVA com Tukey post-hoc foram utilizados conforme apropriado com um nível de significância de $₫=0,05$ e determinado o respetivo valor de eta-parcial quadrado $\left(n^{2} p\right)$. Foram considerados os respetivos limiares de percetibilidade $(\triangle \mathrm{E} 00=0,8)$ e aceitabilidade $(\triangle \mathrm{E} 00=1,8)$. Resultados: $\mathrm{Em}$ todas as técnicas o efeito branqueador foi superior ao limiar de aceitabilidade, sendo os valores de $\triangle \mathrm{E} 00$ significativamente superiores $(\mathrm{P}<0,05)$ no grupo $3(\Delta \mathrm{E} 00=5,2[4,9: 5,4])$. Independentemente da técnica de branqueamento, os dentes de matiz B apresentaram valores médios de $\Delta \mathrm{E} 00$ de 4,5[3,9:5,1] os quais foram significativamente superiores $(\mathrm{P}<0,05)$ quando comparado com o matiz $C$ com um $\triangle \mathrm{E} 00$ médio de 2,7[2,4:3,0]. A análise de variância detetou diferenças significativas $(P<0,05)$ em ambas as variáveis preditivas (técnica de branqueamento e matiz), contudo o tamanho do efeito do matiz (efeito baixo: $\mathrm{n}^{2} \mathrm{p}=0,05$ ) na alteração da cor dentária foi inferior ao da técnica de branqueamento (efeito médio: $\left.n^{2} \mathrm{p}=0,10\right)$. Conclusões: Com base nos resultados obtidos foi possível observar um efeito do matiz na eficácia do branqueamento dentário por diferentes técnicas, sendo a eficácia do branqueamento superior em dentes com matiz B quando comparado ao matiz C.

http://doi.org/10.24873/j.rpemd.2022.01.909

\section{\#053 Difusão pulpar do peróxido de hidrogénio de um produto de branqueamento - estudo in vitro}

Leonor Gonçalves da Silva Casqueiro*, Susana Beatriz Ferreira Dias, Ruben Pereira, João Silveira, António Duarte Mata, Duarte Marques

Grupo de Investigação em Biologia e Bioquímica Oral (GIBBO) - LIBPhys FCT UID/FIS/04559/2013, Faculdade de Medicina Dentária da Universidade de Lisboa

Objetivos: Avaliar a difusão do peróxido de hidrogénio (PH) para a câmara pulpar através dos tecidos dentários, durante a aplicação de um produto de branqueamento com $6 \%$ PH num modelo de pressão pulpar positiva (PPP). Materiais e métodos: Seis molares hígidos do banco de dentes do GI$\mathrm{BBO}$ - UICOB foram selecionados e seccionados horizontalmente 2-3mm abaixo da junção amelo-cementária, com recurso a uma máquina de corte de precisão. O tecido pulpar foi removido com recurso a uma sonda e a coroa foi montada numa placa de policarbonato de forma a simular PPP. A câmara pulpar foi preenchida com solução tampão de acetato $2 \mathrm{M}$ e o volume de cada câmara pulpar foi registado. Foram recolhidas amostras antes (controlo), durante e após o protocolo de branqueamento com $6 \%$ de $\mathrm{PH}$ (VivaStyle Paint On PIus, lvoclar-Vivadent ${ }^{\circledR}$, Liechtenstein), em que se realizaram 6 aplicações de 10 minutos. As amostras foram recolhidas em intervalos de 10 minutos até perfazer $90 \mathrm{mi}$ nutos e subsequentemente analisadas através do método do Leucocristal Violeta. Registou-se a massa de verniz de branqueamento aplicado em cada amostra e a quantidade de $\mathrm{PH}$ que se difundiu para a câmara pulpar. Previamente, foi realizada a titulação do verniz para determinar a concentração de $\mathrm{PH}$ presente no lote utilizado. Os resultados são apresentados como média e intervalo de confiança 95\% (IC), em microgramas de $\mathrm{PH}$ e percentagem de $\mathrm{PH}$ recuperado do conteúdo inicial. Os resultados foram analisados através dos testes one-way ANOVA e Tukey post hoc, com um nível de significância de $\alpha=0,05$. Resultados: Da titulação do 
produto de branqueamento, lote X00724, obteve-se a percentagem média de $6,15 \%$ de $\mathrm{PH}$ presente no verniz. Na avaliação da cinética de difusão do PH para a câmara pulpar foi atingido um máximo de $0,20[0,16-0,25] \mu \mathrm{g} / \mathrm{ml}$ aos $50 \mathrm{minu}-$ tos de aplicação, valor estatisticamente significativo quando comparado com o controlo, sendo este valor inferior ao descrito na literatura como dose citotóxica. A quantidade total de $\mathrm{PH}$ recolhida da câmara pulpar, ao longo de 90 minutos de ensaio, foi de $0,05[0,04-0,07] \mu$, correspondendo a 9,58E-5 [6,33E-5-12,84E-5] \% da quantidade de PH aplicado. Conclusões: A aplicação de um produto de branqueamento com 6\% PH num modelo de pressão pulpar positiva resultou na difusão do $\mathrm{PH}$ até à câmara pulpar. Serão necessários estudos adicionais, de forma a avaliar a possível citotoxicidade pulpar induzida pelas concentrações de PH obtidas neste estudo.

http://doi.org/10.24873/j.rpemd.2022.01.910

\section{\#054 Hipersensibilidade dentária - alterações elementares e moleculares no esmalte}

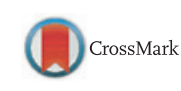

Inês Moniz, Susana Dias, M.R. Correia, Sofia Pessanha, João Silveira*, António Duarte Mata

Departamento de Física - Universidade de Aveiro, LIBPhys FCT UID/FIS/04559/2013, FCT - Universidade Nova de Lisboa, GIBBO - LIBPhys FCT UID/FIS/04559/2013, Faculdade de Medicina Dentária da Universidade de Lisboa

Objetivos: O presente estudo in vitro teve como objetivo analisar modificações elementares e moleculares em amostras de esmalte durante a realização de um tratamento de hipersensibilidade dentária. Para isso, foram utilizadas técnicas espectroscópicas de Fluorescência de raios X Dispersiva em Energia (EDXRF) para obter informação química acerca da composição elementar e ainda a espectroscopia Raman, com o intuito de se analisar o perfil de fosfato. Materiais e métodos: Foram utilizadas vinte amostras de esmalte dentário, provenientes de dentes extraídos por motivos ortodônticos e cirúrgicos. Estas amostras foram divididas aleatoriamente em dois grupos: controlo $(\mathrm{C})$ e teste $(\mathrm{T})$ com recurso a software apropriado.As amostras de ambos os grupos foram escovados com pasta dentifrica Couto. No grupo $\mathrm{T}$ as amostras foram adicionalmente escovadas com a pasta de teste, Biorepair Sensitive Teeth Plus, de forma a respeitar as instruções do fabricante. Entre aplicações, as amostras foram armazenadas em água destilada. As amostras foram analisadas com recurso à técnica de $\mu$-EDXRF e microscopia Raman após 14 e 28 dias de aplicação.As medições de $\mu$-EDXRF foram realizadas com recurso a um espectrómetro M4 Tornado (Bruker, Alemanha), tendo sido realizadas 15 medições por amostra. Os resultados de EDXRF encontram-se expressos em \% de concentração mássica do elementos fósforo, cloro, cálcio, ferro, zinco e estrôncio. As medições de Raman, foram realizadas com recurso a um espectómetro HR800 (Horiba Jobin-Yvon, França) com fonte de laser He-Ne de $632.8 \mathrm{~nm}$. Foram realizadas 10 medições por amostra, com a finalidade de se calcular a razão de despolarização da banda de alongamento simétrico do fosfato
(960 cm-1), expressa em unidades arbitrárias. Resultados: Relativamente à análise elementar não foram registadas diferenças estatisticamente significativas entre os grupos, aos 14 e 28 dias de tratamento.Relativamente à análise molecular, a razão de despolarização dos espectros Raman obtida foi de $0.06 \pm 0.01$ e $0.037 \pm 0.007$ ao fim de 14 e 28 dias, respetivamente. No grupo de teste, as razões de despolarização foram $0.08 \pm 0.01$ ao fim de 14 dias e $0.05 \pm 0.01$ ao fim de 28 dias. A diminuição da razão de despolarização observada é sugestiva de um aumento da mineralização das amostras. Conclusões: A pasta utilizada para o tratamento da hipersensibilidade não resulta na alteração da composição elementar do esmalte. O aumento do grau de mineralização das amostras testadas não é diferente de quando utilizada apenas a pasta de controlo.

http://doi.org/10.24873/j.rpemd.2022.01.911

\#055 Estudo longitudinal de cárie dentária em crianças dos 4 aos 7 anos da Coorte Geração XXI

Cátia Carvalho Silva*, Sandra Gavinha, Rita Rodrigues, Maria da Conceição Manso, Carla Lopes, Paulo Melo

Universidade Fernando Pessoa, Faculdade de Medicina da Universidade do Porto

Objetivos: Investigar a associação prospetiva entre os padrões alimentares e os comportamentos de saúde oral das crianças aos 4 anos de idade e o desenvolvimento de cárie dentária aos 7 anos. Adicionalmente, pretendeu-se avaliar se os comportamentos de risco para a cárie adotados aos 4 anos permaneciam aos 7 anos de idade. Materiais e métodos: A amostra deste estudo foi constituída por 607 crianças da Coorte de nascimento de base populacional Geração XXI, Porto, Portugal. As informações relativas aos hábitos alimentares e comportamentos de saúde oral das crianças foram recolhidas mediante a aplicação de questionários aos responsáveis. A dieta foi avaliada por um questionário de frequência alimentar e três padrões alimentares foram identificados. Nas avaliações da cavidade oral foi utilizado o sistema ICDAS II, por Médicos Dentistas calibrados, para o registo de cárie dentária. Foram definidos 2 outcomes dentários: Desenvolvimento de Cárie Dentária (c3-6pod/C3-6POD >0) e Grave-Desenvolvimento de Cárie Dentária (c3-6pod/ C3-6POD >2) para avaliação da incidência de cárie na fase mista da dentição entre os 4 e os 7 anos de idade. Foram realizadas análises bivariadas e modelos de regressão logística multivariada. Resultados: Entre os 4 e os 7 anos de idade, $51,2 \%$ das crianças apresentavam pelo menos 1 dente com 1 nova lesão de cárie ou 1 dente perdido ou obturado por cárie dentária, enquanto que 27,4\% apresentavam mais de 2 dentes afetados por cárie no mesmo período temporal. As crianças que aos 4 anos de idade apresentavam de forma característica, um padrão alimentar de consumo de 'Alimentos densamente energéticos' (OR=2,19; IC 95\%: 1,41-3,41) e "Snacking" (OR=2,19; IC 95\%: 1,20-4,00), assim como, ingeriam alimentos antes de deitar, após a escovagem dentária (OR=1,77; IC 95\%: 1,15-2,74) apresentavam um risco mais elevado para o Grave-Desenvolvimento de Cárie Dentária 3 